Ann. Biol. anim. Bioch. Biophys., 1979, 19 (1B), 257-266.

\title{
Relations entre l'ingestion d'acides gras à chaîne moyenne par la ratte et la lipogénèse du jeune raton avant le sevrage
}

\author{
par Y. DEMARNE, Marie-José LECOURTIER, J. FLANZY \\ Station de Recherches de Nutrition, I.N.R.A. \\ 78350 Jouy-en-Josas, France.
}

Summary. Relations between rat intake of medium chain fatty acids and rat pup lipogenesis before weaning.

In the rat, prolonged intake of a large quantity of lauric acid (C12:0) before and during pregnancy had no effect on the amount of lipids present in adipose stores at the time of parfurition. However, this medium chain fatty acid was intensely esterified in the triglycerides of the maternal adipose tissues. As compared to a diet rich in long chain fatty acids, the intake of lauric acid during lactation considerably modified mammary gland fatty acid secretion. Lauric acid was esterified in very high proportions (about 35 p. 100 of the total fatty acids) in milk triglycerides, thus considerably increasing its saturated fatty acid content (about 80 p. 100 of the total fatty acids). In these nutritional conditions, the mammary gland could not efficiently regulate the qualitative aspects of fatty acid secretion. However, these large modifications of the milk triglyceride fraction did not change the weight of the pups at weaning and had no effect on their body composition. This would indicate that the growth of the lipid compartment was unchanged. The weight of the liver, its total lipid content and the distributions between neutral lipids and phospholipids were identical to those of the controls. A large amount of the lauric acid ingested by the mother was transferred to the pup in the milk before weaning. A large quantity was found in the body triglycerides $(30 \mathrm{p} .100$ of the total fatty acids) and in neutral liver lipids. It was not incorporated directly into hepatic phospholipids.

\section{Introduction.}

L'animal en croissance ingérant un régime lipidroprive présente un tissu adipeux dont les triglycérides ne contiennent pas d'acides gras de poids moléculaire inférieur à celui de l'acide hexadécanoïque ou palmitique (Demarne et al., 1977a). Au niveau enzymatique, cette observation traduit en fait une incapacité pour le système « acide gras synthétase » du foie et du tissu adipeux à libérer des acides gras de ce type (Martin, Horning et Vagelos, 1961 ; Katiyar et Porter, 1977). Le tissu adipeux possède néanmoins les acyl-transférases capables d'assurer l'estérification de telles molécules, puisque des acides gras à chaîne moyenne (ACM) d'origine exogène ayant de 10 à 14 atomes de carbone sont susceptibles d'être estérifiés dans les triglycérides de réserve (Bach et Metais, 1970). Parmi ceux-ci, l'acide dodécanoíque ou laurique 
(C12:0) est certainement celui qui est le plus intensément incorporé (Demarne et al., 1978a).

Dans la glande mammaire, et contrairement à ce qui est observé au niveau du foie ou du tissu adipeux, des enzymes spécifiques, différentes de la palmitoyl-ACPthioesterase, assurent « in situ » la libération d'une quantité importante d'ACM à partir du système « acide gras synthétase » (Knudsen ef Dils, 1975 ; Knudsen, Clark ef Dils, 1975). Il en résulte que des ACM d'origine endogène, ayant de 8 à 14 atomes de carbone sont estérifiés dans les triglycérides du lait.

Dans des travaux antérieurs, nous avons étudié les effets de l'ingestion prolongée de différents ACM sur les aspects qualitatifs et quantitatifs de la lipogenèse chez le Rat et le Porc après le sevrage (Demarne et al., 1977b, 1978a). L'existence d'une intense activité de synthèse d'ACM dans la glande mammaire fournit une occasion physiologique unique pour rechercher les conséquences d'une interférence entre ACM d'origine alimentaire ef biosynthèse « de novo » de ces molécules. Dans le cadre de cette étude, nous présentons les effets de l'ingestion prolongée d'acide laurique sur les aspects qualitatifs de la biosynthèse des friglycérides du lait chez la Ratte, et ses conséquences sur la lipogenèse du jeune raton avant le sevrage.

\section{Matériel et méthodes.}

\section{Animaux ef régimes.}

Quarante femelles de souche Wistar, d'un pcids moyen d'environ $75 \mathrm{~g}$, sont réparties entre 2 lots $A$ et $B$. Les animaux reçoivent un régime semi-synthétique, contenant 10 p. 100 de lipides et dont la composition a été rapportée antérieurement (Demarne ef al., 1978a). Dans le régime qui est destiné aux animaux du lot $A$, les lipides sont introduits sous forme d'huile de tournesol, tandis que dans celui qui est destiné aux animaux du lot $B$ ils sont introduits sous la forme d'un mélange de tri-dodécanoate ou tri-laurate de glycérol * (Tri C12:0) et d'huile de tournesol $(9: 1 \mathrm{v} / \mathrm{v})$. Les compositions en acides gras des lipides des deux régimes testés sont présentées dans le tableau 2.

Ces femelles sont saillies à un poids moyen de $210 \mathrm{~g}$ et reçoivent pendant toute la durée de leur gestation et de leur lactation le même régime que pendant leur croissance. On considère 10 gestations dans chaque lot. Le poids moyen après la parturition est de $242,0 \pm 8,2 \mathrm{~g}$ dans le lot $\mathrm{A}$ et de $232,4 \pm 7,5 \mathrm{~g}$ dans le lot $\mathrm{B}$ (moyenne \pm écart-type de la moyenne). Le lendemain de leur parturition, 3 femelles dans chaque lot sont sacrifiées de façon à déterminer leur composition corporelle ef la composition en acides gras des triglycérides de réserve en début de lactation. La taille de chacune des 7 portées restantes est égalisée dès le jour de la naissance à 8 ratons chacune. Les mères ef leurs portées sont réparties dans des cages individuelles équipées d'un système d'alimentation qui n'est accessible que pour les mères. Au matin du $8 \mathrm{e}$ jour de lactation $(8 \mathrm{~h}), 8$ ratons provenant de 4 portées différentes sont sacrifiés dans chaque lot. Les contenus stomacaux sont prélevés, puis regroupés par lot. Dans les 2 lots, 3 portées poursuivent leur croissance jusqu'au sevrage à 21 jours. Six ratons sont alors abattus dans chaque lot et le foie est séparé du reste de la carcasse.

\footnotetext{
* Akzo-Chemie France.
} 


\section{Techniques analytiques.}

Tous les animaux sont sacrifiés par décapitation après anesthésie préalable à l'éther. Les foies sont congelés à $-80^{\circ} \mathrm{C}$. Chez les jeunes animaux au sevrage et chez les adultes, le tractus digestif est prélevé ef libéré de son contenu. Les carcasses complètes sont broyées séparément après passage dans l'azote liquide. Les broyats sont ensuite lyophilisés.

Les teneurs en eau des carcasses sont déterminées par pesée avant et après lyophilisation et par déshydratation jusqu'à poids constant d'un échantillon de broyat, dans un four à $70^{\circ} \mathrm{C}$. Les teneurs totales en matières azotées sont obtenues par la méthode de Kjeldahl.

Les lipides du lait, du foie et des carcasses sont extraits en utilisant la méthode proposée par Folch, Lees et Sloane-Stanley (1957). Les triglycérides sont séparés par chromatographie préparative en couche épaisse de gel de silice en utilisant le mélange de migration suivant : hexane ; éther éthylique ; acide formique $(120: 60: 1,5, \mathrm{v} / \mathrm{v})$. Les lipides neutres et les phospholipides hépatiques sont séparés par double passage sur colonne d'acide silicique (Kates, 1972). Les acides gras des lipides totaux corporels, des lipides neutres et des phospholipides hépatiques sont séparés des autres constituants lipidiques par saponification à froid. Ils sont ensuite méthylés à chaud en utilisant le mélange méthanol ; $\mathrm{HCl}(97: 3, \mathrm{v} / \mathrm{v})$. Les acides gras des triglycérides du lait sont transestérifiés (Christie, 1973).

L'analyse des acides gras est réalisée par chromatographie en phase gazeuse en utilisant soit des colonnes classiques, soit des colonnes capillaires. Les techniques analytiques ont été précédemment décrites dans le détail (Flanzy et al., 1976 ; Demarne ef al., 1978a). Toutes les chromatographies sont réalisées plusieurs fois pour chaque échantillon. Compte tenu des très faibles coefficients de variation enregistrés pour chaque concentration d'acide gras et de façon à simplifier les tableaux, nous ne rapporterons dans cette présentation que les valeurs moyennes qui ont été enregistrées.

\section{TABLEAU 1}

Composition corporelle des mères et des jeunes au sevrage (21 j)

\begin{tabular}{lccccc}
\hline $\begin{array}{c}\text { Régimes } \\
\text { maternels }\end{array}$ & Animaux & Poids $(\mathrm{g}) *$ & $\begin{array}{c}\mathrm{H}_{2} \mathrm{O} \\
(\mathrm{p} .100)\end{array}$ & $\begin{array}{c}\mathrm{N} \times 6,25 \\
(\mathrm{p} .100)\end{array}$ & $\begin{array}{c}\text { Lipides } \\
(\mathrm{p} .100)\end{array}$ \\
\hline $\begin{array}{c}\text { Témoin } \\
\text { (lot } \mathrm{A})\end{array}$ & après la parturition $(\mathrm{n}=3) \ldots$ & - & $57,0 \pm 1,9 \mathrm{a}$ & $18,2 \pm 1,6^{\mathrm{a}}$ & $21,4 \pm 1,1^{\mathrm{a}}$ \\
\cline { 2 - 6 } & ratons d̀ 21 $\mathrm{j}(\mathrm{n}=6) \ldots \ldots \ldots$ & $41,0 \pm 1,8$ & $65,6 \pm 0,7^{\mathrm{a}}$ & $13,5 \pm 0,3^{\mathrm{a}}$ & $15,9 \pm 1,0^{\mathrm{a}}$ \\
\hline
\end{tabular}

\begin{tabular}{|c|c|c|c|c|c|}
\hline \multirow{2}{*}{$\begin{array}{l}\text { Tri C12:0 } \\
\text { (lot B) }\end{array}$} & après la parturition $(n=3) \ldots$ & - & $56,3 \pm 1,2_{b}$ & $17,3 \pm 1,1^{b}$ & $23,1 \pm 0,8^{b}$ \\
\hline & ratons d̀ $21 j(n=6)$ & $42,7 \pm 1,1$ & $64,5 \pm 0,8^{b}$ & $14,3 \pm 0,7^{b}$ & $16,6 \pm 0,7^{b}$ \\
\hline
\end{tabular}

* Valeurs moyennes \pm écart-type de la moyenne. Dans une même colonne, les valeurs présentant les mêmes letires sont significativement différentes $(p \leqslant 0,05)$. 


\section{Résultats et discussion.}

Les compositions corporelles des femelles après la parturition sont rapportées sur le tableau 1. On ne note pas de différence en fonction des régimes testés. C'est tout particulièrement le cas pour le compartiment lipidique dont les teneurs en triglycérides augmentent pendant la gestation et diminuent pendant la lactation (Knopp, Herrera et Freinkel, 1970 ; Smith ef Walsh, 1976).

Les compositions corporelles des animaux au sevrage se caractérisent par des teneurs élevées en eau et par des teneurs basses en lipides totaux. L'ingestion prolongée

\section{TABLEAU 2}

Composition en acides gras des lipides des régimes et des triglycérides de réserve des femelles à lo parturition (p. 100 des esters méthyliques dosés)

\begin{tabular}{|c|c|c|c|c|}
\hline $\begin{array}{l}\text { Régimes } \\
\text { maternels }\end{array}$ & & & & \\
\hline Acides gras & Régime * & T. G. (P) & Régime * & T. G. (q) \\
\hline 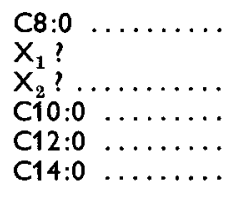 & 0,7 & 1,5 & $\begin{array}{r}\text { tr. } \\
\\
0,4 \\
87,2 \\
1,1\end{array}$ & $\begin{array}{r}34,6 \\
5,1\end{array}$ \\
\hline 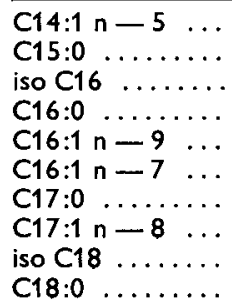 & $\begin{array}{l}\text { tr. } \\
\text { tr. } \\
\text { tr. } \\
7,2 \\
0,5\end{array}$ & $\begin{array}{r}18,8 \\
4,0\end{array}$ & $\begin{array}{l}\text { tr. } \\
1,1 \\
0,5 \\
\operatorname{tr} . \\
\text { tr. } \\
0,5\end{array}$ & $\begin{array}{r}23,5 \\
8,3\end{array}$ \\
\hline $\begin{array}{ll}C 18: 1 n-9 & \ldots \\
C 18: 1 n-7 & \ldots \\
\text { C18:2 } n-6 & \ldots \\
\text { C18:3 } n-6 & \ldots \\
\text { C18:3 } n-3 & \ldots \\
\text { C18:4 } n-3 & \ldots\end{array}$ & $\begin{array}{r}22,8 \\
60,8 \\
0,2\end{array}$ & $\begin{array}{r}27,2 \\
41,5 \\
1,3\end{array}$ & $\begin{array}{l}2,5 \\
4,9 \\
0,1\end{array}$ & $\begin{array}{r}22,1 \\
4,4\end{array}$ \\
\hline $\begin{array}{lll}C 20: 0 & \ldots \ldots \\
\text { C20:1 } n-9 & \ldots \\
\text { C20:2 } n-6 & \ldots \\
\text { C20:4 } n-6 & \ldots \\
\text { C20:4 } n-6 & \ldots \\
\text { C20:5 } n-3 & \ldots \\
\text { C22:1 } n-9 & \ldots \\
\text { C22:5 } n-3 & \ldots \\
\text { C22:6 } n-3 & \ldots\end{array}$ & $\begin{array}{l}0,3 \\
0,4 \\
\\
0,1 \\
\text { tr. }\end{array}$ & & $\begin{array}{c}0,5 \\
0,6 \\
\text { tr. } \\
0,1\end{array}$ & \\
\hline
\end{tabular}

* Analyse réalisée par C. P. G. sur colonne capillaire. 
de trilaurate de glycérol par la mère n'entraîne pas de modifications pour ce qui concerne le poids des jeunes ratons au sevrage ef leur composition corporelle. II apparaît en particulier que la quantité de lipides mis en réserve à 21 jours est identique dans les deux conditions nutritionnelles testées.

Chez les femelles et après la parturition, les compositions en acides gras des triglycérides des réserves corporelles sont essentiellement caractérisées par des concentrations élevées en acide linoléique $(C 18: 2 \omega 6)$ dans le lot $A$ et en acide laurique $(C 12: 0)$ dans le lot B (tabl. 2). Les concentrations en acides linoléique et laurique enregistrées

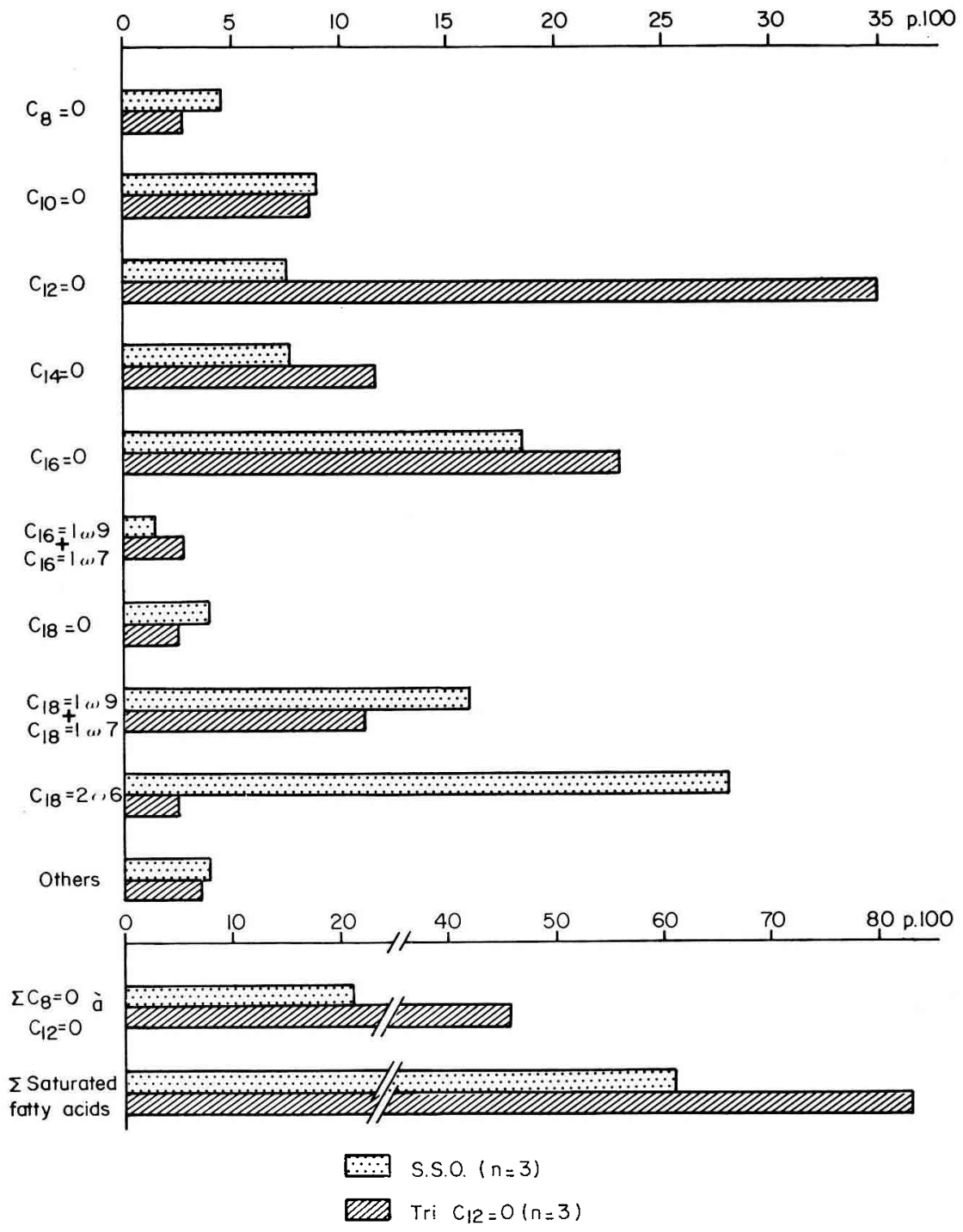

FIG. 1. - Effets de l'ingestion d'huile de tournesol (S.S.O.) ou de trilaurate de glycérol (Tri C12:0) sur la composition en acides gras du lait. 
respectivement dans les lots $A$ et $B$ sont supérieures à celles qui sont observées chez les rats mâles de même poids recevant les mêmes rałions (Demarne, 1978, résultats non publiés). Elles correspondent aux valeurs généralement enregistrées chez des animaux mâles pesant plus de $300 \mathrm{~g}$ (Demarne et al., 1978a). Cette intense estérification d'acides gras d'origine exclusivement exogène chez la jeune femelle en fin de gestation est à rapprocher de l'intense activité lipogénique observée par ailleurs pendant la gestation (Knopp, Herrera et Freinkel, 1970).

Le type de matière grasse ingéré par les mères entraîne de profondes modifications au niveau de la composition en acides gras des triglycérides du lait (fig. 1). Par rapport aux triglycérides isolés à partir du lait des femelles ayant ingéré de l'huile de tournesol (Lot $A$ ), on notera que l'ingestion prolongée de trilaurate de glycérol ne modifie pas les concentrations en acides caprylique et caprique (C8:0 ef C10:0). En revanche, la concentration en acide laurique est 5 fois plus importante. Dans les conditions nutritionnelles éfudiées, les concentrations en acide myristique (C14:0) sont plus importantes dans les triglycérides du lait que dans ceux du tissu adipeux des femelles allaitantes. Cependant, l'ingestion d'acide laurique conduit à une augmentation de la concentration en acide myristique dans les triglycérides secrétés par la mamelle. Au niveau du tissu adipeux, cette augmentation a été antérieurement attribuée à une élongation de l'acide laurique au niveau microsomal (Demarne ef al., 1978a). Dans le lot A, de l'acide myristique est synthétisé « in situ » au niveau de la glande mammaire ef sans apport « exogène » d'acide laurique. Les plus fortes concentrations enregistrées en cas d'ingestion d'acide laurique (Lot $B$ ) peuvent donc résulter à la fois d'une élongation du lauryl-coenzyme $A$ « in situ » ef d'une mobilisation au niveau du tissu adipeux, suivie d'un transfert à la glande mammaire (Smith et Walsh, 1976).

Pour ce qui concerne les acides gras saturés à chaîne longue, des concentrations plus élevées sont enregistrées en cas d'ingestion d'acide laurique. Par rapport au lof témoin, la concentration en acide palmitique $(C 16: 0)$ est alors plus élevée d'environ $30 \mathrm{p}$. 100. L'ensemble des acides gras saturés à point de fusion supérieur à $40^{\circ} \mathrm{C}$ ( $\geqslant \mathrm{C} 12: 0$ ) représente 72 p. 100 des acides gras totaux en cas d'ingestion de trilaurate de glycérol contre seulement 37 p. 100 dans le lot de référence $A$.

L'ingestion de trilaurate de glycérol conduit à une diminution des concentrations en acides gras monoinsaturés (C16:1 et C18:1). La concentration en acide linoléique

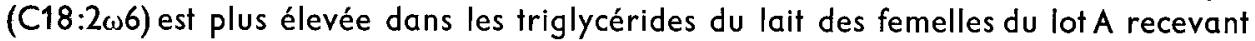
de l'huile de tournesol. Il est à noter cependant que, même si l'apport alimentaire de cet acide gras est peu important (Lot B), sa concentration dans les triglycérides du lait reste 2 fois plus faible que dans les triglycérides des graisses de réserve.

Chez le raton sevré, les triglycérides de réserve sont caractérisés par des compositions en acides gras intermédiaires entre celles des triglycérides du lait ef celles des triglycérides des tissus adipeux maternels (fig. 2). L'acide laurique, qui ne représente que 5 p. 100 des acides gras totaux dans le lot $A$, est intensément estérifié chez les animaux du lot $B$ ( 30 p. 100 des acides gras totaux). Comme l'acide linoléique dans le lot $A$, l'acide laurique est donc intensément transféré au jeune par l'intermédiaire des triglycérides du lait directement, ou indirectement après avoir été estérifié dans les graisses corporelles maternelles et avoir été mobilisé pendant la lactation. Comme chez l'adulte, lacide laurique est fortement incorporé dans les réserves adipeuses avant le 
sevrage. Récemment on a émis une hypothèse selon laquelle l'accumulation de cet acide gras serait associée à une saturation des enzymes microsomales qui assurent l'élongation des acides gras (Dermane ef al., 1978a). En cas d'ingestion de trilaurate de glycérol par la mère, les triglycérides des tissus adipeux du jeune raton au sevrage contiennent plus de 70 p. 100 d'acides gras saturés ( $\geqslant C 12: 0)$.

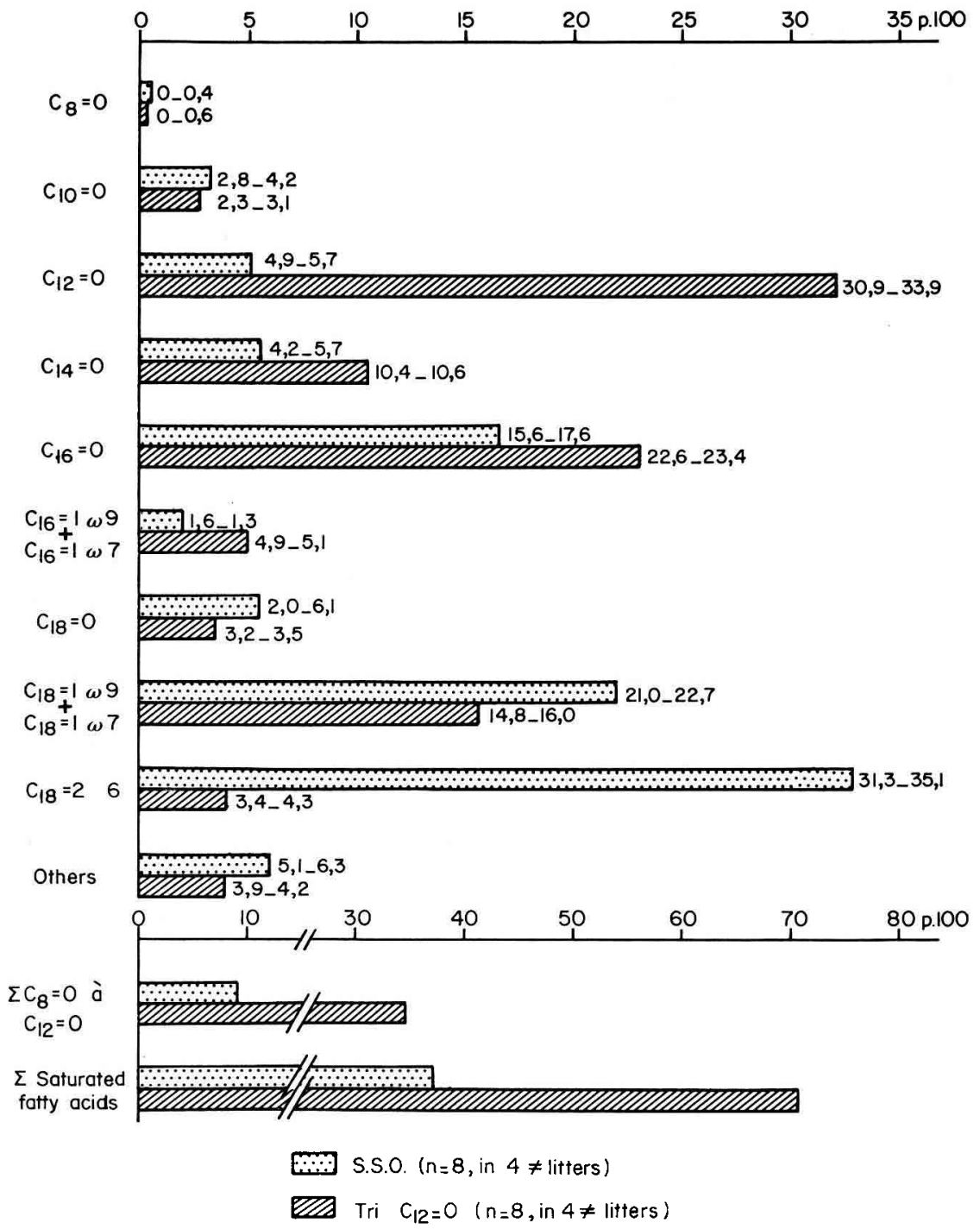

FIG. 2. - Composition en acides gras des triglycérides de réserve du jeune raton au sevrage (21 j) (S.S.O. : huile de tournesol ; Tri C12:0 : trilaurate de glycérol).

Comme on peut le voir sur le tableau 3, le type de lipide ingéré par la mère n'entraîne pas de modifications au niveau du poids du foie des jeunes au sevrage. Il en 
est de même pour les teneurs en lipides totaux et les proportions respectives de lipides neutres et de phospholipides.

TABLEAU 3

Poids du foie et teneurs en lipides au sevrage (21 i)

\begin{tabular}{lcccc}
\hline Régime maternel & Poids (g) & $\begin{array}{c}\text { Lipides } \\
(\text { p. 100) }\end{array}$ & $\begin{array}{c}\text { Lipides neutres } \\
\text { (p. 100) }\end{array}$ & $\begin{array}{c}\text { Phospho-lipides } \\
\text { (p. 100) }\end{array}$ \\
\hline Témoin (lot A) $\ldots$ & $1,6 \pm 0,2$ & $4,1 \pm 0,3$ & 24,2 & 75,5 \\
\hline Tri C12:0 (lot B) $\ldots$ & $1,8 \pm 0,1$ & $5,0 \pm 0,4$ & 29,3 & 71,3 \\
\hline
\end{tabular}

* Valeurs moyennes \pm écart-type de la moyenne.

Les compositions en acides gras des lipides neutres et des phospholipides hépatiques sont présentées sur le tableau 4. Comme cela a déjà été rapporté par le cas de l'animal adulte (Demarne et al., 1978b), les lipides neutres hépatiques présentent une composition en acides gras très proche de celle qui est observée au niveau des triglycérides du tissu adipeux quand l'alimentation assure un apport important d'acides gras à chaîne longue. En revanche, en cas d'un apport exogène important d'ACM, les lipides neutres présentent une composition bien particulière caractérisée surtout par une concentration en acide laurique $(\mathrm{C} 12: 0) 3$ fois plus faible que dans les triglycérides

TABLEAU 4

Composition en acides gras des fractions lipides neutres (LN) ef phospholipides (PL) du foie du sevrage à 21 (p. 100 des esters méthyliques dosés)

\begin{tabular}{|c|c|c|c|c|}
\hline \multirow{2}{*}{$\frac{\text { Régime maternel }}{\text { Acides gras }}$} & \multicolumn{2}{|c|}{ Témoin (lot A) } & \multicolumn{2}{|c|}{$\begin{array}{l}\text { Tri C12:0 } \\
\text { (lot B) }\end{array}$} \\
\hline & $\mathrm{LN} *$ & $\mathrm{PL} *$ & $\mathrm{LN} *$ & $\mathrm{PL}^{*}$ \\
\hline $\begin{array}{l}\mathrm{C} 10: 0 \ldots \ldots \ldots \ldots \\
\mathrm{C} 12: 0 \\
\mathrm{C} 14: 0 \\
\mathrm{C} 16: 0 \ldots \ldots \ldots \ldots\end{array}$ & $\begin{array}{r}\overline{0,7} \\
1,1 \\
18,9\end{array}$ & $\begin{array}{c}\overline{-} \\
1,3 \\
16,7\end{array}$ & $\begin{array}{r}1,2 \\
11,1 \\
7,0 \\
23,1\end{array}$ & $\begin{array}{c}- \\
0,7 \\
23,3\end{array}$ \\
\hline C16:1 $\}+\begin{array}{l}n-7 \ldots \\
n-9\end{array}$ & 0,9 & 0,7 & 4,1 & 2,5 \\
\hline$C 18: 0 \quad \ldots \ldots \ldots \ldots$ & 4,2 & 21,6 & 5,0 & 23,7 \\
\hline C18:1 $\}+\begin{array}{l}n-7 \ldots \\
n-9 . .\end{array}$ & 26,2 & 6,3 & 26,3 & 8,2 \\
\hline$C 18: 2 n-6 \quad \ldots \ldots$ & 34,1 & 14,6 & 5,2 & 6,8 \\
\hline $\begin{array}{c}C 18: 3 n-3 \\
\left.\begin{array}{l}\dagger \\
C 20: 1 n-9\end{array}\right\} \ldots . .\end{array}$ & 0,8 & 1,0 & 0,9 & 0,5 \\
\hline $\begin{array}{l}C 20: 4 n-6 \\
\text { C20:5n-3 }\end{array}$ & $\begin{array}{l}\frac{8,7}{\overline{4}} \\
-1\end{array}$ & $\begin{array}{r}22,4 \\
0,4 \\
1,2 \\
13,2\end{array}$ & $\begin{array}{r}\overline{-} \\
4,6 \\
11,2\end{array}$ & $\begin{array}{r}11,3 \\
1,9 \\
1,5 \\
18,2\end{array}$ \\
\hline
\end{tabular}

* Dans toutes les fractions on a enregistré la présence de C20:3n - 9 à l'état de traces $(0,1$ p. 100). 
corporels. Le foie est un site d'oxydation particulièrement actif pour les acides gras, et les ACM sont plus rapidement et plus intensément oxydés que les acides gras à chaîne longue (Bach, Metais et Warter, 1968 ; Metais, Bach et Warter, 1967). Une oxydation hépatique plus rapide de l'acide laurique pourrait, tout au moins en partie, expliquer cette différence.

Dans les deux lots, on remarque également que les acides gras polyinsaturés à chaîne longue ( $\omega 6$ et $\omega 3$ ) sont présents à des concentrations élevées dans les lipides neutres hépatiques des jeunes ratons au sevrage. Ces observations, caractéristiques du jeune animal, confirment les résultats antérieurement présentés et discutés par Sinclair et Crawford (1972) et Sinclair (1974).

L'acide laurique n'est pas directement estérifié dans les phospholipides hépatiques, même quand l'apport exogène est très important. Ceci confirme les données enregistrées précédemment chez l'animal sevré (Bezard et Monneret-Boquillon, 1966 ; Demarne et al., 1978b). Les différences enregistrées au niveau des compositions en acides gras des phospholipides concernent surtout les acides gras polyinsaturés à chaîne longue. Il apparaît que ces différences sont certainement en relation avec la concentration beaucoup plus élevée en acide linoléique observée dans le lait du lot $A$. Avant le sevrage, l'absorption d'une quantité plus importante d'acide linoléique conduirait ainsi à l'estérification d'une quantité 2 fois plus importante d'acide linoléique $(\mathrm{C} 18: 2 \omega 6)$ et arachidonique $(\mathrm{C} 20: 4 \omega 6)$ dans les phospholipides hépatiques du jeune au sevrage.

\section{Conclusions.}

La présence d'une quantité importante d'acide laurique (C12:0) dans l'alimentation de la femelle gestante est sans effet sur les quantités de lipides présents dans les réserves adipeuses au moment de la parturition. Cependant, cet ACM est intensément estérifié dans les triglycérides des dépôts adipeux maternels. Par rapport à une alimentation riche en acides gras à chaîne longue, l'ingestion d'acide laurique conduit à de profondes modifications de la secrétion d'acides gras par la mamelle. L'acide laurique est estérifié dans de très fortes proportions (environ 35 p. 100 des AGT) dans les triglycérides du lait, ce qui augmente considérablement la teneur en acides gras saturés (environ 80 p. 100 des AGT). La glande mammaire, dans ces conditions nutritionnelles, n'est donc pas capable de réguler avec efficacité les aspects qualitatifs de la secrétion des acides gras.

Néanmoins, ces modifications importantes de la fraction triglycérides du lait ne changent pas le poids des jeunes au sevrage et sont sans effet sur leur composition corporelle, ce qui laisse supposer, en particulier, que la croissance du compartiment lipidique n'est pas modifiée. Par rapport aux animaux témoins, le poids du foie, sa teneur en lipides totaux et les répartitions entre lipides neutres et phospholipides sont identiques. Par l'intermédiaire du lait, l'acide laurique ingéré par la mère est transféré en quantité importante au jeune avant le sevrage. On le retrouve en forte proportion dans les triglycérides corporels (30 p. 100 des AGT) et dans les lipides neutres hépatiques. II n'est pas directement incorporé dans les phospholipides hépatiques. 


\section{Références}

BACH A., METAIS P., 1970. Graisses à chaînes courtes et moyennes. Aspects physiologiques, biochimiques, nutritionnels et thérapeutiques. Ann. Nutr. Alim., 24, 75-144.

BACH A., METAIS P., WARTER J., 1968. Comparaison par l'étude du $14-\mathrm{CO}_{2}$ de l'air expiré de l'utilisation des graisses à acides gras longs, moyens et courts. C. R. Soc. Biol., 162, 247-251.

BEZARD J., MONNERET-BOQUILLON M., 1966. Captation par le foie en perfusion des acides gras à chaînes courtes et moyennes. Arch. Sci. physiol., 20, 359-378.

CHRISTIE W. W., 1973. Lipid analysis. Isolation, separation, identification and structural analysis of lipids, Pergamon Press Oxford, $338 \mathrm{p}$.

DEMARNE Y., EPO N., FLANZY J., LECOURTIER M. J., 1978o. Comparison of long ferm lipogenic effects of two middle-chain triglycerides (Tri C8:0 and Tri C12:0) on liver lipids in the growing rat. Arch. int. Physiol. Bioch. (sous presse).

DEMARNE Y., EPO N., FLANZY J., LECOURTIER M. J., 1978b. Effects of two different medium chain triglycerides (Tri C8:0 and Tri C12:0) on liver lipids in the growing rat. Arch. int. Physiol. Bioch. (sous presse).

DEMARNE Y., PERAZA-CASTRO C., HENRY Y., FLANZY J., 1977a. Effets des lipides alimentaires sur les aspects qualitatifs de la lipogenèse chez le rat et le porc en croissance. Ann. Biol. anim. Bioch. Biophys., 17, 875-886.

DEMARNE Y., PERAZA-CASTRO C. E., HENRY Y., FLANZY J., 1977b. Effects on lipid fatty acid composition in different adipose deposits of pig ingesting glycerol tri-dodecanoate (Tri C12:0). Ann. Biol. onim. Bioch. Biophys., 17, 137-146.

FLANZY J., BOUDON M., LEGER C., PIHET J., 1976. Application of Carbowax-20M as an opentubular liquid-phase in analysis of nutrionnally important fats or oils. J. chromatog. Sci., 14, 17-24.

FOLCH J., LEES M., SLOANE-STANLEY G. H., 1957. A simple method for the isolation and purification of total lipids from tissues. J. biol. Chem., 226, 497-509.

KATES M., 1972. Techniques of lipidology. Isolation, analysis and identification of lipids, North Holland/American Elsevier, Amsterdam, 267-610.

KATIYAR S. S., PORTER J. W., 1977. Mechanism of fatty acid synthesis. Life Sci., 20, 737-760.

KNOPP R. H., HERRERA E., FREINKEL N., 1970. Carbohydrate metabolism in pregnancy. VIII. Metabolism of adipose tissue isolated from fed and fasted pregnant rats during late gestation. J. clin. Invest., 49, 1438-1446.

KNUDSEN J., CLARK S., DILS R., 1975. Acyl-COA hydrolase(s) in rabbit mammary gland which control the chain length of fatty acids synthesized. Biochem. biophys. Res. Comm., 65, 921-926.

KNUDSEN J., DILS R., 1975. Partial purification from rabbit mammary gland of a factor which controls the chain length of fatty acid synthesized. Biochem. biophys. Res. Comm., 63, 780-785.

MARTIN D. B., HORNING M. G., VAGELOS P. R., 1961. Fatty-acid synthesis in adipose tissue. I. Purification and properties of a long chain fatty acid-synthesizing system. J. biol. Chem., 236, 663-668.

METAIS P., BACH A., WARTER J., 1967. Comparaison par l'étude du 14-CO $\mathrm{CO}_{2}$ de l'air expiré de l'utilisation des graisses à acides gras longs, moyens ou courts. C. R. Soc. Biol., 161, 1372-1376.

SINCLAIR A. J., 1974. Fatty acid composition of liver lipids during development of rat. Lipids, 9, 809. 818.

SINCLAIR A. J., CRAWFORD M. A., 1972. The incorporation of linolenic acid and docosahexaenoic acid into liver and brain lipids of developing rats. F.E.B.S. Lett., 26, 127-129.

SMITH R. W., WALSH A., 1976. Effect of lactation on lipolysis in rat adipose tissue. Lipids, 11, 418-420. 